\title{
LA TEORÍA DE LOS ESTADIOS DE LA LENGUA Y LA GRADACIÓN DE LA COMPLEJIDAD LINGÜÍSTICA
}

\author{
CARMEn Conti JimÉnEZ \\ Universidad de Jaén
}

\begin{abstract}
RESUMEN
Parte de los trabajos recientes que estudian la complejidad lingüística relacionan la presencia de morfología flexiva con un mayor grado de complejidad. Así sucede, por ejemplo, en el estudio de Dahl 2004 sobre la evolución de la lengua y en el de McWhorter 2001 sobre la simplicidad de las lenguas criollas. En este artículo, se pretende demostrar que el origen de esta relación se encuentra en las propiedades que se atribuyen desde el siglo XIX en adelante a los tipos aislante, aglutinante y flexivo dentro de la teoría de los estadios de la lengua, que pondera el tipo flexivo por haber alcanzado un mayor grado de perfección. El último cometido de este artículo es mostrar, al margen de la teoría de los estadios de la lengua y con la ayuda de algunas nociones de las Ciencias de la Complejidad, que carecemos del metalenguaje, del método y del aparato empírico necesario para probar que el tipo flexivo posee una mayor complejidad algorítmica y una mayor complejidad evolutiva que los demás tipos morfosintácticos.
\end{abstract}

Palabras clave: Ciencias de la Complejidad, complejidad evolutiva, complejidad lingüística, evolución lingüística, teoría de los estadios de la lengua, tipo aislante, tipo flexivo.

\begin{abstract}
Some recent works on linguistic complexity relate the occurrence of inflectional morphology with a higher complexity. That is the case of the study by Dahl 2004 on the evolution of language and the one by McWhorter 2001 on the simplicity of Creole languages, among others. In this paper, we intent to demonstrate that the origin of this relationship may be found in the properties associated, since the nineteenth century to our days, with the isolating, agglutinative, and inflectional types in the theory of the states of the language, in which the inflectional type is overestimated because of its perfection. Lastly, we will show -leaving aside the theory of the states of the language and taking into account some concepts from the Sciences of Complexity - that we lack the appropriate language, method and empirical basis to demonstrate that the inflectional type is algorithmically and evolutionarily more complex.

Key Words: evolutionary complexity, inflectional type, isolating type, linguistic complexity, linguistic evolution, Sciences of Complexity, theory of the states of the language.
\end{abstract}




\section{INTRODUCCIÓN}

El estudio del cambio lingüístico en tanto que proceso evolutivo que tiende al progreso y contribuye a la mejora de las lenguas recibió una considerable atención a lo largo del siglo XIX. Es en este siglo cuando se forja la teoría de los estadios de la lengua, en la que se defiende la existencia de una escala evolutiva entre los tipos aislante, aglutinante y flexivo $-\mathrm{o}$, en su variante extendida, entre los tipos aislante, aglutinante, flexivo y analítico-.

Como intentaré demostrar a lo largo de este trabajo, la idea de que cada uno de estos tipos se corresponde con un grado diferente de maduración y perfección de la lengua dio lugar, junto a otras ideas, a la asociación del tipo flexivo con una mayor complejidad lingüística.

En este artículo me propongo, primero, rastrear en algunos textos de los siglos XVIII y XIX los orígenes de esta asociación de ideas y, segundo, ilustrar cómo dicha asociación se ha perpetuado hasta nuestros días en algunos trabajos sobre el cambio lingüístico y el origen de las lenguas.

Por otro lado, pretendo que el lector tenga la oportunidad de juzgar críticamente los criterios que han llevado a ciertos estudiosos a postular que el tipo flexivo se encuentra en el último estadio de la escala evolutiva de las lenguas y es, como resultado del paso del tiempo, el culmen de la maduración del sistema.

Finalmente, mostraré las razones por las que considero poco probable que se pueda llegar a demostrar alguna vez que el tipo flexivo es más complejo que otros tipos morfosintácticos.

Este trabajo consta de cuatro secciones, aparte de la introducción y el apartado bibliográfico. En la sección 2, se presenta la teoría de los estadios de la lengua y se muestran sus posibles vínculos con la medición de la complejidad lingüística. En 3, se describe cómo esta teoría ha condicionado nuestra idea de lo que es una lengua simple y una lengua compleja. Para ello, se revisarán dos propuestas recientes: el principio de maduración de Dahl 2004 y la hipótesis de la simplicidad de las lenguas criollas. En el apartado 4, se plantean las dificultades teóricas, empíricas y metodológicas que supone relacionar el tipo flexivo con el paso del tiempo y con una mayor complejidad algorítmica y evolutiva. En la última sección se presentan las conclusiones del trabajo.

\section{LA TEORÍA DE LOS ESTADIOS DE LA LENGUA Y LA COMPLEJIDAD LINGÜÍSTICA}

En la teoría de los estadios de la lengua se defiende la existencia de una escala evolutiva entre distintos tipos morfosintácticos. Esta teoría, que tiene sus antecedentes en el siglo XVIII y se forja en el siglo XIX, se 
emplea aún hoy en Lingüística. El éxito de este constructo radica en su fácil adaptación a ideas y teorías de distintas etapas del pensamiento europeo, como el progreso social, la relación pensamiento-lenguaje, la transmutación de las especies, etcétera.

El germen de la teoría de los estadios de la lengua se encuentra en el reconocimiento de al menos dos tipos morfosintácticos a los que se asigna un determinado valor. Por ejemplo, en el Ensayo práctico de las lenguas (1787), el padre Hervás y Panduro observa que el chino, pese a ser una «lengua rústica», es hablada por un pueblo civilizado, y el araucano, pese a ser una "lengua artificiosa», es hablada por un pueblo bárbaro (Hervás y Panduro 1991, p. 15) ${ }^{1}$. A nadie se le escapa que la calificación de lengua rústica para el chino y de lengua artificiosa para el araucano oculta dos tipos morfosintácticos diferentes: uno sin apenas morfología -como el chino- y otro con una presencia abrumadora de morfemas -como ocurre con las lenguas araucanas, que son polisintéticas-.

Algo antes, el eminente economista y sociólogo Adam Smith había redactado el ensayo Consideraciones sobre la primera formación de las lenguas y el diferente genio de las lenguas originales y compuestas (1761), que aparecerá publicado en lo sucesivo junto a su Teoría de los sentimientos morales. En este texto, Adam Smith reconoce dos tipos de lenguas con diferentes grados de complejidad. Así, describe las declinaciones y las conjugaciones de las lenguas clásicas como estrategias complejas, frente al carácter simple de las expresiones que denomina «compuestas», que ilustra con la lengua inglesa. Véase la siguiente selección de pasajes²:

Si las declinaciones de las lenguas clásicas son complejas, lo son aún más sus conjugaciones. Y la complejidad de la segunda se basa en el mismo principio de la primera: la dificultad de formar, en los inicios de la lengua, términos abstractos y generales (Smith 1853, p. 523).

[...] este cambio [de morfemas de caso a preposiciones] es, sin duda, una simplificación de la lengua en lo relativo a los rudimentos y principios. Introduce, en lugar de una gran variación de declinaciones, una declinación universal, que es idéntica en todas las palabras, sea cual fuere su género, número o terminación (Smith 1853, p. 531).

El ensayo de Adam Smith se cita por lo general en los estudios actuales como propulsor del denominado principio de compensación (véase, por ejemplo, Smith y Tapia 2002). En efecto, Adam Smith es el primer

\footnotetext{
${ }^{1}$ El debate en torno a la relación entre grado de perfección de una lengua y progreso social es propio del siglo XVIII y pervivirá hasta el XIX. Por lo general, los religiosos desaprueban esta relación.

${ }^{2}$ La traducción del inglés al español es mía.
} 
pensador que defiende que las lenguas que son simples en ciertos aspectos son complejas en otros. En particular, comenta ${ }^{3}$ :

En general, se puede proponer la máxima de que, cuanto más simple es una lengua en su composición, más compleja debe ser en sus declinaciones y conjugaciones; y, por el contrario, cuanto más simple es en sus declinaciones y conjugaciones, más compleja debe ser en su composición (Smith 1853, p. 532).

La máxima de Adam Smith es sorprendentemente novedosa para la época. Sin embargo, el ensayo de este pensador no debe entenderse como una apología de la igualdad entre las lenguas. Muy al contrario, el escocés opina que las lenguas modernas han experimentado un proceso de simplificación que las hace a todas luces más imperfectas que las lenguas clásicas, tal y como se colige a partir de los siguientes pasajes ${ }^{4}$ :

Primero, las lenguas que experimentan esta simplificación son más prolijas, llegando a ser necesarias numerosas palabras para expresar lo que antes podía ser expresado mediante una palabra. Así, las palabras Dei y Deo del latín muestran suficientemente, sin ningún añadido, la relación que se establece entre el objeto signado y el resto de objetos expresados por otras palabras en la oración. Mas, para expresar la misma relación, en inglés [...] debemos usar, al menos, dos palabras, y decir of God, to God.

[...] En segundo lugar, esta simplificación de los principios de las lenguas las hace menos agradables al oído.

[...] En tercer lugar, esta simplificación no solo hace los sonidos de esta lengua menos agradables al oído, sino que nos impide situar los sonidos como nos plazca, en el modo que fuera más agradable. Fija muchas palabras en una determinada posición, pese a que colocarlas en otra las haría más bellas (Smith 1853, pp. 535-536).

Adam Smith considera, por tanto, que la simplificación de las lenguas modernas ha originado un aumento innecesario en el número de palabras, ha dado lugar a cierta cacofonía o rudeza fónica en la lengua y, en el caso del inglés, a un orden fijo de constituyentes que, en opinión del autor, restringe la variación estilística.

La creencia de que las lenguas presentan tipos estructurales distintos con un grado de perfección variable se consolida con el ensayo de Schlegel Sobre la lengua y la sabiduría de los indios (1808) y con el ensayo póstumo de Humboldt Sobre la diversidad de la estructura del lenguaje humano y su influencia sobre el desarrollo espiritual de la humanidad (1836) ${ }^{5}$. En

3 La traducción es mía.

${ }^{4}$ La traducción es mía.

${ }^{5}$ En el ensayo Sobre la lengua y la sabiduría de los indios (1808), Schlegel reconoce también la perfección de las lenguas flexivas. Sin embargo, ve en las lenguas indoeuropeas modernas como el inglés un mayor grado de eficacia y conveniencia por haber simplificado la gramática para un mejor uso general. Esto es, Schlegel defiende la superioridad de las lenguas analíticas. 
particular, Humboldt elabora en el citado ensayo la famosa clasificación tipológica que distingue entre lenguas aislantes, aglutinantes y flexivas y establece, además, una correspondencia entre cada uno de esos tipos y un determinado grado de perfección y ajuste entre lenguaje y pensamiento, cuyo exponente máximo es el tipo flexivo.

La interpretación teleológica de la evolución de los tipos lingüísticos recibirá el tratamiento de teoría de los estadios de la lengua en la obra de Sayce titulada Principios de filología comparada (1875), quien describe así dicha teoría ${ }^{6}$ :

Se nos dice a menudo que uno de los logros de la ciencia del lenguaje ha sido demostrar un desarrollo continuo y regular en la historia del habla: primero, un estadio aislante, o periodo de las raíces, cuando la posición de la palabra denotaba el significado de una oración sin la ayuda de ningún signo relacional (auxiliar); luego, un estadio aglutinante, momento en el que se añadieron esas marcas auxiliares, manteniendo cada una de ellas, eso sí, un significado pleno; y finalmente, un estadio flexivo, cuando las marcas auxiliares pierden su significado independiente y dan lugar a multitud de signos inseparables. El estadio último presenta una tendencia posterior al análisis: la flexión se rompe y los compuestos en decadencia se utilizan, como en inglés, para expresar de forma independiente, con la ayuda de la posición, las distintas relaciones en las que se resuelve la oración. El periodo analítico difiere del aislante en que, en el último, cada raíz es el germen que contiene en sí mismo cada modo y relación, mientras que, en el primero, el germen ha sido roto en sus elementos, y estos son representados por palabras que son, cada una de ellas, reliquias de una era anterior de tipo flexivo. Se supone que los tres estadios responden a la vida individual y solitaria que, se imagina, llevó el primer hombre [...] hasta la vida tribal y familiar de los nómadas y, finalmente, la vida social del ciudadano civilizado. El chino se toma como ejemplo del primer tipo; el turco, del segundo; y el sánscrito, del tercero (Sayce 1875, pp. 132-133).

El texto de Sayce ilustra a la perfección cómo la teoría de los estadios de la lengua aglutinó finalmente las escalas de Schlegel y Humboldt, la teoría del progreso social y la teoría de la transmutación de las especies.

La correlación entre tipo morfosintáctico y complejidad lingüística se sobreentiende a partir de los rasgos que describen cada uno de esos $\operatorname{tipos}^{7}$. El hecho de que el tipo flexivo se asocie con la ruptura de la univocidad del signo lingüístico, con el último eslabón de la evolución lingüística y, sobre todo, con la presencia de irregularidades contribuirá

\footnotetext{
${ }^{6}$ La traducción del inglés es mía. Sayce, que se opone a la teoría darwinista, defiende la existencia de distintas razas y distintas lenguas desde el origen, y no como resultado de la variación.

7 Por ejemplo, Schlegel tilda de simple a la lengua que denomina indio -sánscrito- por ser regular (Schlegel 1849, p. 445).
} 
sin duda a su caracterización como el tipo más complejo. En el lado opuesto de la balanza, se sitúan las lenguas aislantes y, en algunos trabajos, como el de Jespersen 1922, también las lenguas analíticas. Como veremos en el siguiente apartado, estos rasgos se utilizan aún hoy como diagnósticos de una mayor o menor complejidad lingüística, lo que pone de manifiesto, en mi opinión, la enorme trascendencia de la teoría de los estadios de la lengua.

\section{LA TEORÍA DE LOS ESTADIOS DE LA LENGUA Y LA MEDICIÓN DE LA COMPLEJIDAD LINGÜÍSTICA HOY}

La complejidad lingüística es un tema de cierta actualidad que ha despertado el interés de distintos especialistas pertenecientes a diferentes ámbitos de estudio. Los conceptos «simple»y «complejo» aparecen en trabajos en los que se aborda bien la recreación de la primera lengua hablada por el ser humano, bien el cambio lingüístico, bien la medición de la complejidad entre las lenguas modernas. En estos trabajos, prevalece la idea de que los primeros estadios de la lengua son más simples que los estadios ulteriores, pues la complejidad lingüística se contempla como una propiedad gradual y graduable que aumenta con el paso del tiempo.

El estudio de la complejidad lingüística sigue estando condicionado, sin embargo, por ideas de origen decimonónico, como la teoría de los estadios de la lengua que acabo de presentar. Como mostraré en los siguientes apartados, gran parte de los trabajos recientes que abordan el estudio de la complejidad lingüística atribuyen un mayor grado de complejidad a la presencia de morfología flexiva que a la de otros tipos morfosintácticos.

Con este fin, revisaré, primero, el denominado principio de maduración de la lengua de Dahl 2004 y, segundo, la hipótesis de la simplicidad de las lenguas criollas, pues ambos casos mantienen un vínculo estrecho con la teoría de los estadios.

\subsection{El principio de maduración de Dahl 2004}

Una de las hipótesis que mejor dejan entrever la influencia de la teoría de los estadios de la lengua es la de Dahl 2004. De acuerdo con este autor, la presencia de ciertas construcciones en las lenguas es muestra de una mayor complejidad evolutiva del sistema como resultado del paso del tiempo. En particular, Dahl 2004, p. 105, explica':

\footnotetext{
${ }^{8}$ La traducción es mía.
} 
[...] Consideremos las marcas de plural en lenguas como el tok pisin y el inglés. En tok pisin, ol, que deriva del inglés all 'todo', se usa regularmente como marcador de plural en los nombres. La historia de este marcador, como muestra el propio tok pisin, no puede ser muy larga. Además, la marcación del plural en tok pisin carece de las siguientes propiedades atestiguadas en inglés para la categoría de número:

(i) marcación flexiva;

(ii) numerosos casos de idiosincrasia léxica (plurales irregulares);

(iii) empleo obligatorio de plurales incluso en contextos en los que la marca de número es informativamente redundante, p. ej. después de cuantificador (many books 'muchos libros');

(iv) concordancia sintáctica (verbos, pronombres demostrativos);

(v) existencia de pluralia tantum (palabras que solo aparecen en plural: scissors 'tijeras', trousers 'pantalones').

Parece razonable pensar que un sistema como el del inglés y otras lenguas indoeuropeas solo puede surgir después de un desarrollo histórico de una profundidad significativa -que supone una serie de estadios intermedios-, mientras que los sistemas tempranos se parecen más al tok pisin (aunque la fuente última del morfema de plural sea diferente). Por tanto, la categoría de número en inglés posee un mayor grado de complejidad evolutiva.

Nótese que Dahl atribuye un mayor grado de complejidad a ciertas características que ya se vinculaban en el siglo XIX con las lenguas consideradas complejas: presencia de morfología flexiva (así en el punto [i]); presencia de irregularidad en los paradigmas flexivos (así en el punto [ii] y [v]); y exceso de marcación (así en el punto [iii]).

La aportación más significativa de este autor es el desarrollo del denominado principio de maduración o madurez de la lengua. Dahl 2004, p. 105, opina que hay ciertos fenómenos lingüísticos que deben tener una prehistoria, es decir, que no pueden carecer de cierta complejidad evolutiva. Esos fenómenos son, en términos del autor, maduros y se caracterizan por lo siguiente:

\section{Definición de maduro}

$x$ es un fenómeno maduro si y solo si hay algún fenómeno identificable y no universal o un conjunto restringido de fenómenos $y$ tales que, para cualquier lengua L, si existe $x$ en L, hay algún ancestro L' de L tal que L' tiene $y$ pero no $x$ (Dahl 2004, p. 105).

Más adelante, Dahl 2004, pp. 111-112, concluye que el fenómeno maduro por excelencia es la morfología flexiva y, dentro de la sintaxis, la concordancia, en la que incluye las marcas de caso.

Para Dahl 2004, por otro lado, la madurez de las formas lingüísticas se alcanza gradualmente, a lo largo del tiempo, de modo que la presencia de fenómenos maduros supone la existencia de estadios previos con una serie de características. En particular, Dahl 2004, pp. 106-107, pro- 
pone la escala de maduración libre $>$ perifrástico $>$ afijal $>$ fusionante para dar cuenta del hecho de que las estructuras lingüísticas evolucionan desde un primer estadio, caracterizado por la presencia de formas libres, hasta un último estadio de morfología flexiva que, según el autor, es la culminación del proceso de maduración del sistema y signo de una mayor complejidad evolutiva. La fase perifrástica y la afijal son estadios intermedios en la escala de cambio.

En resumen, el principio de maduración supone dos cosas que relacionan la propuesta de Dahl 2004 con la teoría de los estadios: primero, que las fases iniciales de la lengua están constituidas por formas atómicas no descomponibles -caracterización muy próxima a la idea tradicional del tipo aislante y del periodo de las raíces-; y segundo, que la morfología fusionante, que engloba la flexión, es producto del paso del tiempo y no puede documentarse en los primeros momentos de la génesis de la lengua.

\subsection{La hipótesis de la simplicidad de las lenguas criollas}

En la sección anterior, pudimos ver que Dahl 2004 comparaba la supuesta complejidad evolutiva del inglés con la también supuesta simplicidad del tok pisin, una lengua criolla de base inglesa hablada en Papúa-Nueva Guinea. Las lenguas criollas han despertado un enorme interés entre los lingüistas por tratarse de sistemas que, al ser de formación relativamente reciente e ilustrar un pretendido primer estadio evolutivo, bien pudieran arrojar algo de luz al estudio y comprensión de la ontogenia de las lenguas modernas.

Como veremos en breve, la ausencia de determinadas unidades y relaciones en esas lenguas ha llevado a distintos estudiosos a tildarlas de simples y, a veces, de lenguas imperfectas, lo que ha oscurecido, en mi opinión, el verdadero interés de estas lenguas para la tipología ${ }^{9}$. En este apartado pretendo demostrar que la supuesta simplicidad de las lenguas criollas es un espejismo inspirado en parte por la teoría decimonónica de los estadios de la lengua. Mas, antes de abordar este asunto, presentaré qué se entiende por lengua criolla y en qué condiciones sociales y lingüísticas surge.

La génesis de las lenguas criollas tuvo lugar en zonas de intercambio comercial o laboral muy intenso en las que, además, se dio la circunstancia de que los miembros de la comunidad hablaban lenguas diferentes y pertenecían a distintos estratos sociales. En muchos casos, como sucedía con las plantaciones de esclavos en las antiguas colonias, la len-

\footnotetext{
${ }^{9}$ Degraff 2004 ha criticado duramente el supuesto carácter excepcional de las lenguas criollas frente a otras lenguas naturales.
} 
gua o las lenguas de los amos actuaron a manera de superestrato lingüístico, mientras que la lengua o las lenguas de los esclavos lo hicieron como sustrato. En estas condiciones de vida, se espera que los esclavos y empleados aprendan a defenderse en la lengua de la clase dominante, y no al contrario. Además, la lengua de la clase dominante solía emplearse en determinadas circunstancias como interlingua entre trabajadores de procedencias muy variadas que hablaban lenguas distintas.

En la actualidad, se estima que existen unas ochenta y seis lenguas criollas, cuya lengua dadora o de superestrato puede ser el inglés, el portugués, el suahelí, el árabe, etc. ${ }^{10}$. El chabacano en Filipinas y el palenquero en Colombia son lenguas criollas que tienen como base léxica el español. El palenquero se formó en el siglo XVII a partir de un sustrato afroportugués y el chabacano en los siglos XVI y XVII a partir de un sustrato austronésico ${ }^{11}$.

Gran parte de los especialistas reconoce que, pese a surgir en zonas geográficas muy alejadas y tener una base lingüística diferente, las lenguas criollas convergen en un mismo prototipo. En efecto, estas lenguas se caracterizan en conjunto por carecer de palabras funcionales (como las adposiciones), por no tener flexión y por presentar un mismo orden de constituyentes (que es el de sujeto+verbo+objeto) ${ }^{12}$.

Como se observa en el ejemplo de (1), el papamiento -de base romance, probablemente portuguesa- expresa nuestro sintagma preposicional en casa mediante un sintagma nominal sin preposición ni marca morfológica de caso (p. ej. kas) ${ }^{13}$ :

(1) Papamiento (Maurer 1998, p. 180)

\begin{tabular}{|c|c|c|c|c|}
\hline$[\ldots]$ & $\begin{array}{l}M i \\
1 \mathrm{SG}\end{array}$ & $\begin{array}{l}\text { no } \\
\text { NEG }\end{array}$ & $\begin{array}{l}t a \\
\text { PRESENTE }\end{array}$ & $\begin{array}{l}\text { queda } \\
\text { quedarse }\end{array}$ \\
\hline
\end{tabular}

Por otro lado, parece que las lenguas criollas carecen de morfemas flexivos para la expresión del tiempo y el aspecto verbales. En su lugar, emplean partículas verbales, como se observa en el ejemplo (1) citado anteriormente, donde $t a$ marca tiempo presente. Se ha señalado, asimismo, que las lenguas criollas carecen de morfemas de género y número en el sustantivo. Así sucede en chabacano, lengua en la que el

${ }^{10}$ Es el número de lenguas criollas que aparece en la versión web de Ethnologue, que está disponible en <http://www.ethnologue.com/show_family.asp?subid=90083> (página consultada el 8 de abril de 2009).

11 Remito a Klee y Lynch 2009, quienes ofrecen una visión de conjunto bastante clara sobre las lenguas criollas de base española.

12 Para las características de las lenguas criollas, véanse, entre otros, Appel y Muysken 1987 y Field 2004.

13 NEG es abreviatura de negación y SG, de singular. 
plural de los sustantivos se expresa mediante una forma libre (p. ej. manga en manga bahay 'casas', Grant 2007, p. 187).

Se han dado distintas explicaciones a la convergencia de las lenguas criollas en un mismo prototipo, algunas de ellas en el intento de demostrar que estas lenguas reflejan las primeras fases (simples, imperfectas) de formación de un sistema lingüístico. Así, se considera que las lenguas criollas son el resultado de un aprendizaje imperfecto de una segunda lengua (Valdman 1981); o sistemas que, al no haber evolucionado lo suficiente, presentan gramáticas simples o con escasa complejidad evolutiva (McWhorter 2001, Dahl 2004).

En conclusión, los rasgos que caracterizan las lenguas criollas, como la ausencia de morfología y la transparencia semántica, se han interpretado como signos de una menor complejidad del sistema. A esta visión subyace la teoría de los estadios de la lengua, pues las primeras fases de existencia de la lengua se asocian con estadios sin flexión.

4. AlgunAS REFLEXIONES EN TORNO A LA ASOCIACIÓN ENTRE TIPO FLEXIVO, COMPLEJIDAD LINGÜÍSTICA Y PASO DEL TIEMPO

En los apartados anteriores, he querido mostrar que parte de los trabajos recientes que abordan el estudio de la complejidad lingüística relacionan la presencia de morfología flexiva con un mayor grado de complejidad por influjo de la teoría de los estadios de la lengua.

En este apartado, espero poder demostrar, partiendo de algunos parámetros utilizados para la medición de la complejidad en las lenguas artificiales y en el marco de las Ciencias de la Complejidad, que el tipo flexivo no es más complejo que otros o, al menos, que la relación entre complejidad y flexión es difícil de demostrar.

Dicho esto, es necesario aclarar que todas las lenguas son sistemas igualmente complejos por ser producto de seres con una misma facultad y capacidad intelectiva ${ }^{14}$. La lengua es compleja porque posee un conjunto de unidades que se relacionan mediante reglas de combinatoria; es algorítmica (se pueden generar infinidad de estructuras a partir de un número reducido de unidades y reglas); mantiene un equilibrio entre orden y desorden (caos) para ser informativa; es críptica y tiene profundidad (es decir, las lenguas modernas existen desde hace miles de años $)^{15}$. En suma, la lengua es compleja por distintas razones y, en con-

${ }_{14}$ Chomsky 2002, p. 89, apunta la posibilidad de que el sistema computacional recursivo del órgano de la lengua esté fijado y determinado -como expresión de los genes-, junto con la estructura básica de los posibles ítems léxicos.

${ }^{15}$ Los tres primeros criterios son algunos de los que utiliza Edmonds 1999 para calibrar la complejidad de las lenguas artificiales. Los dos últimos proceden de Gell-Mann 1995 y serán tratados con detalle en el siguiente apartado. 
secuencia, lo es en distintos sentidos. Si estuviéramos dispuestos a medir el grado de complejidad de una lengua frente a otra, deberíamos tener en cuenta, por tanto, varios parámetros de análisis: podríamos comparar el número de unidades de una y otra lengua o la presencia de una mayor o menor irregularidad, por ejemplo. En lo que atañe a las posibles variaciones de la complejidad en virtud de la presencia de distintos tipos morfosintácticos, parece de mayor interés centrarse en aquellos parámetros que afectan al número y la clase de reglas que describen una determinada estructura morfosintáctica (complejidad algorítmica), así como en aquellos otros que guardan relación con el paso del tiempo por el sistema, como la profundidad (complejidad evolutiva).

Por este motivo, los siguientes apartados se centran en el estudio de la posible relación entre el tipo flexivo y el aumento de la complejidad algorítmica y evolutiva de la lengua.

\subsection{Tipo flexivo y complejidad algoritmica}

El empleo de un tipo morfosintáctico u otro para expresar los mismos contenidos funcionales supone una variación en la longitud del mensaje lingüístico, más comprimido en las estructuras sintácticas de tipo flexivo y menos en las de tipo aislante. Para ilustrar mis palabras, permítaseme ofrecer un par de ejemplos. Como vimos en otro lugar de este texto, el papiamento, lengua criolla de tipo aislante, emplea una forma libre para codificar el tiempo y el aspecto verbales, y otra forma distinta para marcar la persona gramatical del sujeto, que generalmente aparece como elemento autónomo o palabra. Así se observa en el ejemplo ya citado Mi no ta queda kas «No quiero quedarme en casa» (Maurer 1998, p. 180), donde $m i$ es primera persona del singular y desempeña la función de sujeto y $t a$, la marca de tiempo y aspecto del verbo. Por su parte, el latín clásico, que responde al patrón flexivo típico, puede llegar a utilizar una única forma ligada para expresar el tiempo y el modo verbales y la persona y el número del sujeto, como sucede, por ejemplo, en el verbo laudo 'elogio', donde la vocal final informa de que el sujeto es primera persona del singular, el verbo está en tiempo presente y el modo es indicativo.

Si reparamos en los ejemplos, la longitud del mensaje de la oración del papiamento es algo mayor que la del latín. Por tanto, son indicios de una mayor longitud del mensaje lingüístico:

(i) cualquier relación entre forma y contenido que no se corresponda con una forma para varios contenidos gramaticales;

(ii) el empleo de varias formas para un mismo contenido gramatical. 
Por el contrario, es signo de una menor longitud el empleo de una forma para varios contenidos gramaticales.

Supongamos que pudiera haber distintos grados de complejidad algorítmica si relacionamos la longitud del mensaje lingüístico con el número y calidad de las reglas que se necesitan para la descripción de dicho mensaje: ¿es el tipo flexivo, asociado a mensajes de menor longitud, resultado de un mayor número de reglas? ¿Presenta, pues, el tipo flexivo un mayor grado de complejidad algorítmica? Mi respuesta, que argumentaré en los párrafos siguientes, es que carecemos del metalenguaje apropiado para medir las posibles variaciones de la complejidad algorítmica.

En primer lugar, debemos tener en cuenta que la naturaleza de las reglas que describen el mensaje en una lengua como el papamiento es diferente de la de las reglas que describen un mensaje como el del latín: las primeras son reglas de formación de constituyentes y las segundas son reglas de formación de palabras. De partida, por tanto, parece poco adecuado establecer una comparación entre elementos (en este caso, reglas) de naturaleza dispar que pertenecen a distintos niveles gramaticales.

Si a esto añadimos que las reglas que describen la formación de estructuras lingüísticas se pueden recoger mediante mensajes de longitud variable (que dependerán, sobre todo, del metalenguaje empleado) y pueden ser mayores o menores en número en virtud del modelo teórico en el que enmarquemos nuestra propuesta, resulta francamente difícil, si no imposible, medir la complejidad algorítmica de los distintos tipos morfosintácticos. En efecto, el número de reglas y su longitud varían notablemente si explicamos las construcciones dentro de la Gramática Relacional, de la Gramática Generativa o la Gramática del Papel y la Referencia, por citar algunos modelos.

En conclusión, la existencia de variación algorítmica asociada al empleo de estrategias morfosintácticas diferentes es indemostrable. La multiplicidad de metalenguajes y modelos que explican el lenguaje humano no permiten realizar un estudio comparativo uniforme y objetivo.

Sí podemos admitir con cierta rotundidad, sin embargo, que todo mensaje lingüístico es en términos generales un mensaje comprimido, esto es, es un mensaje que posee una longitud menor que la longitud del mensaje que lo describe y lo explica. Para ilustrar que, en efecto, el mensaje lingüístico está comprimido voy a tomar dos diagramas de representación que pertenecen a diferentes modelos teóricos: el modelo funcional de la Gramática del Papel y la Referencia (en el diagrama de 2) y el modelo generativista del programa de Principios y Parámetros (en el diagrama de 3). El diagrama de (2) representa los distintos pasos y operaciones que, de acuerdo con el modelo de la Gramática del Papel y la Referencia, se han dado en inglés para formar la oración Scully did not show Mulder the photo at the office yesterday «Ayer, Scully no le 
mostró a Mulder la foto en la oficina» (véase Van Valin 2007, p. 33). El diagrama de (3) ilustra, de acuerdo con la propuesta de Baker 1996, p. 427, cómo se genera la oración del mojave wahakenatarakwétar «Me cortó el pan» ${ }^{16}$.

Al margen de los modelos teóricos y de los metalenguajes empleados, en los dos modos de representar y explicar sendas estructuras lingüísticas, las oraciones propiamente dichas son mensajes con una longitud inferior a la del mensaje propuesto en cada uno de los modelos. En cierto sentido, pues, los mensajes lingüísticos están más comprimidos que el mensaje que los describe y los explica. Pues bien, un mensaje comprimido es aquel que, para ser descrito y explicado, necesita de una longitud de mensaje considerablemente superior ${ }^{17}$.

Como señala Gell-Mann 1995, el hecho de que determinados mensajes estén comprimidos puede llevarnos a pensar que la complejidad efectiva del sistema que los genera es baja y, por tanto, simple. En particular, Gell-Mann 1995, p. 105, se refiere a mensajes altamente com-

(2) Diagrama de Van Valin 2007, p. 33

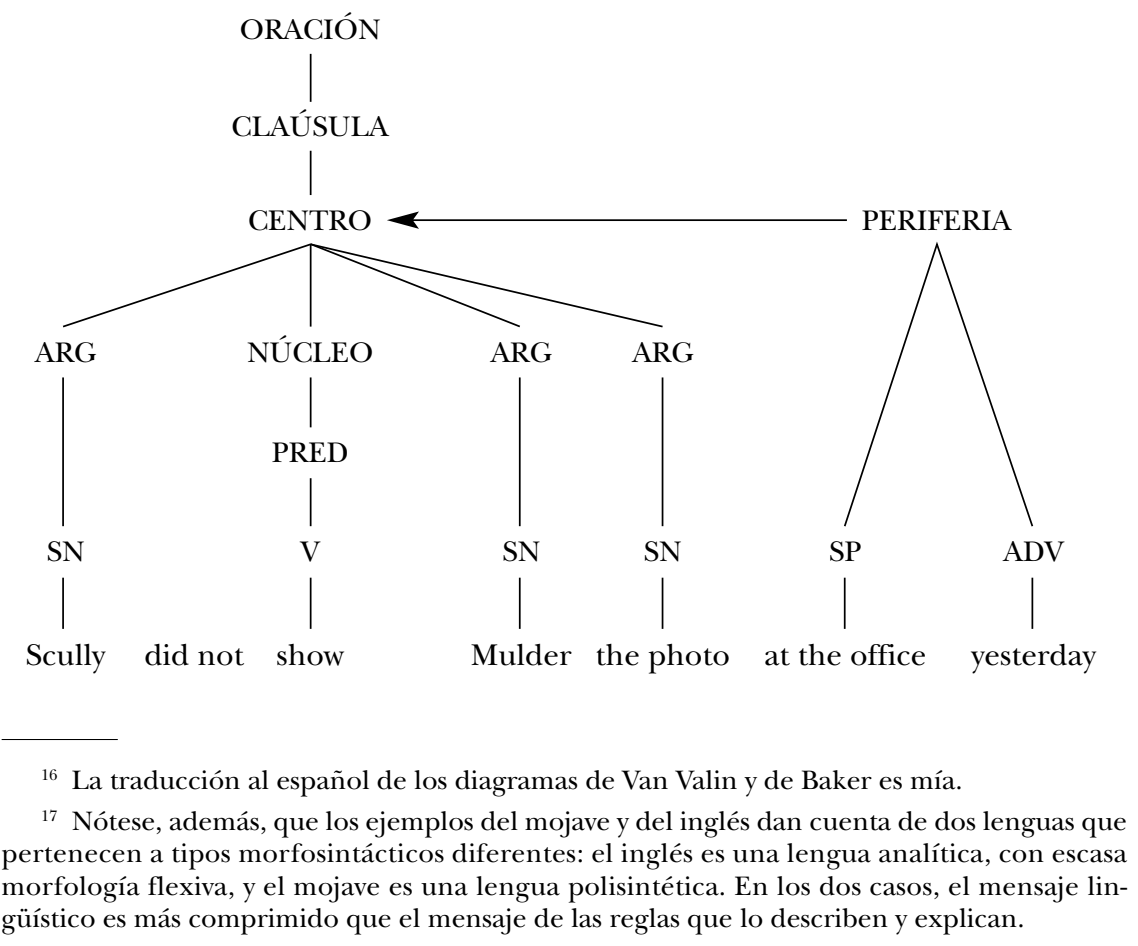


(3) Diagrama de Baker 1996, p. 427

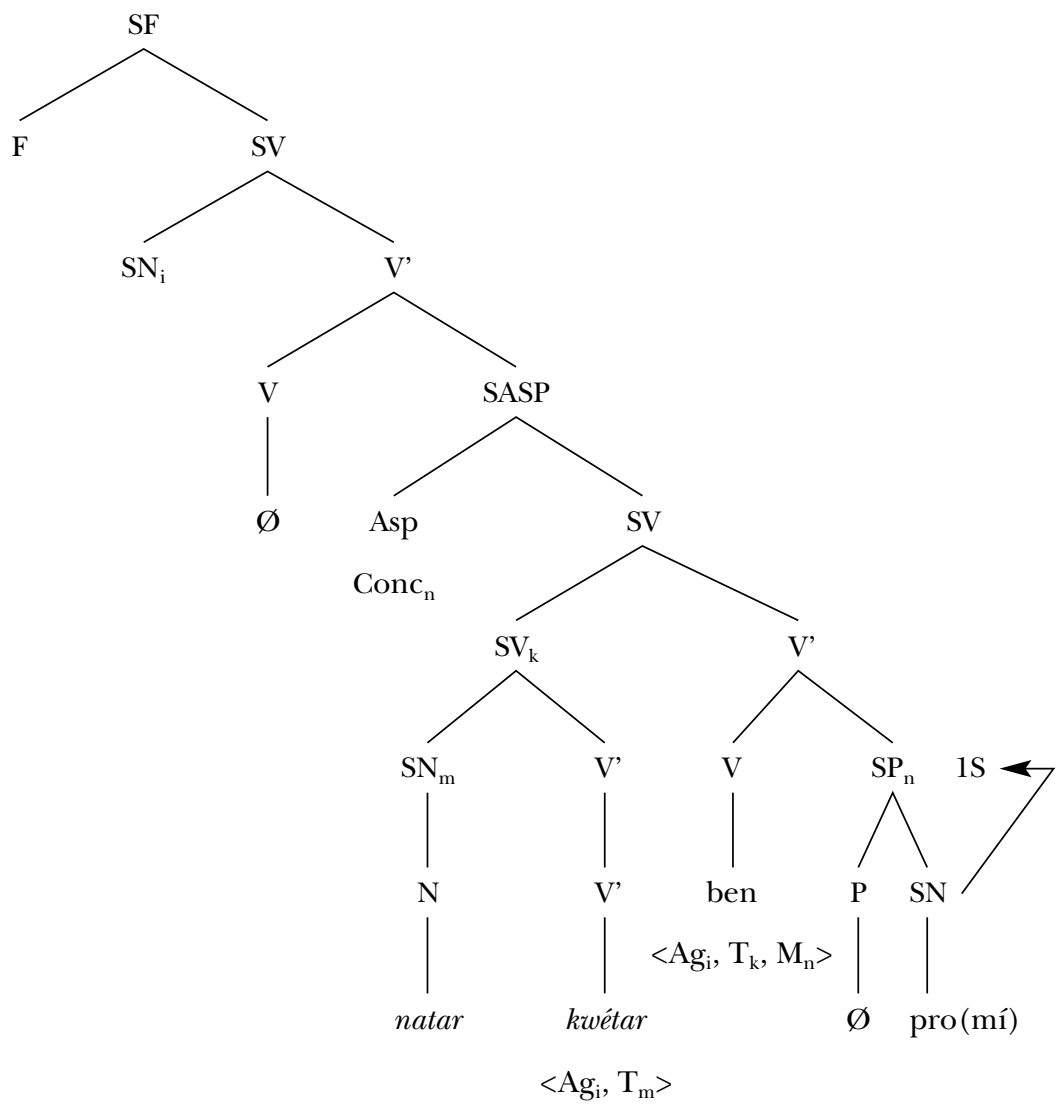

primidos como el de la teoría de la relatividad general de Einstein: $\mathrm{G}_{\mu \nu}=8 \pi \mathrm{K} T_{\mu \nu}$. En casos de compresión como estos, Gell-Mann 1995 señala que se han de tener en cuenta dos factores antes de llegar a la conclusión de que se trata de un sistema con una complejidad efectiva baja: la profundidad del sistema y el carácter críptico del esquema.

Pues bien, la profundidad, de acuerdo con Gell-Mann 1995, pp. 118119, se refiere a aspectos como: ¿cuánto cuesta pasar del esquema altamente comprimido a una descripción completamente desarrollada del propio sistema o de sus regularidades?; mientras que el carácter críptico se refiere a cosas como ¿cuánto cuesta, partiendo del sistema, comprimir su descripción (o una descripción de sus regularidades) en un programa o esquema? 
El carácter críptico de ciertos esquemas comprimidos se refiere, de acuerdo con Gell-Mann 1995, p. 123, al tiempo que emplean los teóricos para descifrar una cadena de bits. Aplicado a las lenguas, el carácter críptico tendría que ver con el tiempo que nos lleva descifrar una lengua ignota, por ejemplo, o bien con el tiempo empleado en realizar la gramática de una lengua. Las lenguas son sistemas que generan mensajes crípticos, ya que descifrar una lengua desconocida o describir los esquemas regulares de una gramática dada supone un trabajo de años o, en algunos casos, una misión imposible.

Según Gell-Mann 1995, p. 121, la profundidad es en cierto modo la medida del tiempo que tardará en generarse el mensaje. En la naturaleza, el hecho de que un sistema posea una profundidad muy grande nos indica que ha tardado mucho tiempo en evolucionar, o bien que tiene su origen en otro sistema al cual le ha llevado mucho tiempo evolucionar a su vez. La profundidad no tiene que ver en el caso de la lengua con el tiempo de generación del mensaje o con el esfuerzo que empleamos en su producción: la profundidad en las lenguas guarda relación con su configuración en tanto que sistemas complejos que existen en el tiempo.

Como dije más arriba, las estructuras lingüísticas presentan un grado de compresión mayor que el mensaje de las reglas que las describen, de lo que se deduce que las lenguas modernas, en su conjunto y en el marco del tiempo de nuestra especie, poseen una profundidad significativa. Esto implica, además, que cualquier lengua creada en el periodo actual en un entorno lingüístico dado es compleja desde su origen porque la profundidad de los sistemas lingüísticos se transmite generación tras generación.

Las lenguas modernas en su conjunto, por tanto, son sistemas complejos que tienen una profundidad considerable y están altamente comprimidos. Ahora bien, ¿existe alguna correlación entre el tipo de longitud de los mensajes de una lengua dada y la profundidad en un sentido restringido, esto es, entre el tipo de mensaje, el número de reglas que lo describen y el paso del tiempo sobre ese sistema específico? ¿Son los mensajes producidos por tipos fusionantes resultado del paso del tiempo frente a los mensajes menos comprimidos, como los del tipo aislante? En otras palabras, ¿es demostrable que las formas libres están en el origen de la evolución lingüística y las formas fusionantes, al final, como propone Dahl 2004?

Lo que defenderé aquí es que la estrategia flexiva -que, como hemos visto, no implica una mayor complejidad algorítmica- no siempre es resultado de procesos de cambio que requieren la existencia de estadios intermedios previos y largos periodos de tiempo para constituirse, lo que significa que el tipo flexivo no siempre es indicio de que el sistema posee una mayor complejidad evolutiva. 


\subsection{Tipo flexivo y complejidad evolutiva}

La idea de que el tipo flexivo se origina a partir de otros tipos morfosintácticos que experimentan cambios graduales guarda relación con el hecho de que los lingüistas hayan podido atestiguar para ciertos morfemas fusionantes de distintas lenguas un origen en formas léxicas que se han gramaticalizado. La gramaticalización da cuenta de una cadena de cambio lingüístico que arranca en una primera forma léxica -o con cierta carga léxica- y culmina en una forma fusionante con valor funcional. Entre una y otra forma, se reconocen distintos estadios intermedios (generalmente, auxiliares u otra clase de formas cuasi ligadas, resultado de la desemantización y erosión fónica de la forma original) que permiten reconstruir una cadena de cambio gradual. Como la gramaticalización es un proceso que requiere el paso del tiempo, parece lógico que los afijos flexivos, último estadio del cambio, se interpreten como formas maduras a las que les ha llevado largo tiempo constituirse.

Si todos los morfemas fusionantes de las lenguas cuyo tipo morfosintáctico es flexivo se originaran a partir de procesos como la gramaticalización, se debería admitir que las lenguas flexivas son sistemas por los que ha tenido que pasar mucho tiempo y en los que se puede reconocer una complejidad evolutiva considerable, como apunta Dahl 2004.

La creación de morfemas flexivos, sin embargo, no responde siempre a un proceso de cambio gradual que acontece a lo largo de un extenso periodo de tiempo. Jespersen 1922 observa que algunos morfemas flexivos de las lenguas indoeuropeas han surgido a raíz del fenómeno que denomina secretion, según el cual «una porción de una palabra indivisible adquiere un valor gramatical que no tenía en origen, y que pasa a sentirse después como algo que se ha añadido a la palabra» (Jespersen 1969 , p. 384 ${ }^{18}$. De acuerdo con el autor, la porción de la palabra que termina por desgajarse gana entidad como morfema cuando comienza a adjuntarse a otras palabras que no poseían ese elemento.

Por ejemplo, Jespersen 1969, p. 385, apunta que el origen del morfema de plural -en del inglés, que se conserva hoy tan solo en las formas oxen 'bueyes' y children 'niños', se encuentra en una consonante nasal que, en antiguo inglés, era parte del radical del sustantivo. En particular, señala que la terminación - $n$ era parte del lexema de los sustantivos que en antiguo inglés pertenecían a la denominada declinación en $-n$. El sustantivo $o x$, cuyo nominativo singular en inglés antiguo era oxa, presentaba un radical en - $n$ para el resto de los casos tanto en singular como en plural. Según Jespersen 1969, p. 385, en inglés medio, la forma $-n$ desaparece de la declinación en singular (p. ej. se crean

\footnotetext{
${ }^{18}$ La traducción es mía.
} 
singulares analógicos, como el genitivo oxes) y se conserva tan solo en las formas de plural. Esto sucede con otros sustantivos que pertenecían a la declinación en - $n$ del antiguo inglés, como sterren 'estrellas', eyen 'ojos', etc. A partir del momento en que $-n$ se interpreta como marca vinculada a la expresión de un plural, su uso se extiende por analogía a otros sustantivos que originalmente no pertenecían a la declinación en $-n$.

Cabe suponer que, de haberse producido, fenómenos como el descrito por Jespersen 1922 hubieron de ser en cierto sentido abruptos o requerir un espacio de tiempo inferior a los procesos episódicos como la gramaticalización ${ }^{19}$. De admitir esto, se podría postular que al menos ciertos tipos flexivos se han formado en un tiempo menor del que se les supone tradicionalmente y, por tanto, que no todos ellos presentan una complejidad evolutiva alta. Por otro lado, resulta muy difícil demostrar en qué grado fenómenos como la secreción pudieron contribuir a la formación de los paradigmas flexivos de las lenguas, ya que se trata de fenómenos de difícil reconstrucción por carecer de un origen léxico. De hecho, Jespersen 1969, p. 386, concluye a este respecto:

Dado que esta distribución irregular o arbitraria se encuentra de forma similar en casi todos los finales flexivos de los estadios más antiguos de las lenguas que pertenecen a nuestra familia, es más que probable que muchos de esos finales que no podemos retrotraer a sus orígenes surgieran mediante secreción o mediante un proceso similar, más que a través de la coalescencia de palabras independientes o raíces.

Desafortunadamente, la vía de análisis propuesta por Jespersen no ha tenido continuadores en la lingüística posterior, pese a haber abierto una puerta a la explicación del tipo flexivo al margen de la teoría de los estadios de la lengua.

\section{Conclusiones}

Parte de los trabajos recientes que abordan el estudio de la complejidad lingüística relacionan la presencia de morfología flexiva con un mayor grado de complejidad sin ofrecer argumentos sólidos.

La falta de argumentación revela en cierto sentido que la atribución de una mayor complejidad al tipo flexivo entronca con una larga

\footnotetext{
19 En la biología moderna, la divergencia de las especies se explica en ocasiones mediante cambios abruptos. La noción de cambio abrupto tiene cada vez más aplicaciones a la explicación de la ontogenia de las lenguas. Por ejemplo, Logan 2006 entiende que el nacimiento de la primera lengua hablada por el ser humano tuvo que ser abrupta, esto es, sin que existiera previamente una protolengua verbal rudimentaria -sin sintaxis-.
} 
tradición lingüística que ha perpetuado una serie de ideas que se dan por probadas. En este artículo, he intentado demostrar que, en efecto, el origen de esta asociación se encuentra en lo que he denominado la teoría de los estadios de la lengua, o escala evolutiva entre distintos tipos morfosintácticos. Esta teoría, que tiene sus antecedentes en el siglo XVIII y se forja en el siglo XIX, condiciona en la actualidad gran parte de los parámetros de medición de la complejidad, como he querido mostrar a través del principio de maduración de la lengua (Dahl 2004) y la hipótesis de la simplicidad de las lenguas criollas.

Una vez descritos los orígenes de la asociación entre tipo flexivo y complejidad -y una vez descartada también la pertinencia de esta asociación-, he querido demostrar con parámetros utilizados para la medición de la complejidad en las lenguas artificiales y en el marco de las Ciencias de la Complejidad que el tipo flexivo no es más complejo que otros. Para ello, me he centrado en el análisis de las posibles variaciones de la complejidad algorítmica -número de reglas y longitud de esas reglas- y de la complejidad evolutiva -efecto de la maduración del sistema como consecuencia del paso del tiempo-. He concluido, primero, que la existencia de distintos metalenguajes y modelos teóricos para la descripción de la lengua impide una medición objetiva de la complejidad algorítmica; y, segundo, que el posible origen de ciertos morfemas flexivos a partir de fenómenos abruptos indica que la presencia de flexión no siempre es signo de una mayor complejidad evolutiva. En suma, resulta indemostrable que el tipo flexivo sea producto de un mayor número de operaciones algorítmicas como resultado del paso del tiempo en el sistema.

\section{REFERENCIAS BIBLIOGRÁFICAS}

Appel, R. y MuYsken, P. (1987): Language contact and bilingualism, Londres, Edward Arnold.

BAKER, M. C. (1996): The polysynthesis parameter, Nueva York, Oxford, Oxford University Press.

Chomsky, N. (2002): «Language and the brain», en Belletti, A. y Rizzi, L. (eds.), On nature and language, Cambridge, Cambridge University Press, pp. 61-91.

DAHL, Ö. (2004): The growth and maintenance of linguistic complexity, Ámsterdam, Filadelfia, John Benjamins.

DeGrafF, M. (2004): «Against creole exceptionalism (Redux)», Language 80, 4, pp. 834-839.

Edmonds, B. (1999): Syntactic measures of complexity, Tesis doctoral, Departamento de Filosofía, Universidad de Manchester. Disponible en <http://scholar. google.es/scholar?q=Syntactic+Measures+of+Complexity\&hl=es\&as_sdt= 0\&as_vis=1\&oi=scholart $>$ (última consulta: 19-04-2010). 
FIELD, F. (2004): «Second language acquisition in creole genesis. The role of processability», en Escure, G. y Schevegler, A. (eds.), Creoles, contact, and language change: Linguistics and social implications, Filadelfia, John Benjamins, pp. 127-160.

Gell-Mann, M. (1995): El quark y el jaguar. Aventuras en lo simple y lo complejo, Barcelona, Tusquets (trad. de García, A. y Pastor, R.).

Grant, A. P. (2007): «Some aspects of NPs in Mindanao chabacano: Structural and historical considerations», en Baptista, M. y Guéron, J. (eds.), Noun phrases in creole languages. A multi-faceted approach, Ámsterdam, Filadelfia, John Benjamins, pp. 173-204.

Hervás y Panduro, L. (1787/1991): Vocabulario poligloto y Saggio pratico delle lingue, Madrid, Sociedad General Española de Librería (intr. de Breva-Claramonte, M. y Sarmiento, R.).

Humboldt, W. von (1836/1972): Linguistic variability and intelectual development, Philadelphia, University of Pennsylvania Press (trad. inglesa de Buck, G. C. y Raven, F. A.).

JESPERSEN, O. (1922/1969): Language: its nature, development and origin, Londres, Georges Allen \& Unwin Ltd.

KleE, C. A. y Lynch, A. (2009): El español en contacto con otras lenguas, Washington, Georgetwon University Press.

LOGAN, R. K. (2006): "The extended mind model of the origin of languages and cultures», en Gontier, N., van Bendegem, J. P. y Aerts, D. (eds.), Evolutionary epistemology, language and culture. A non-adaptationist, systems theoretical approach, Dordrecht, Springer, pp. 149-167.

MAurer, Ph. (1998): «El papiamento de Curazao», en Perl, M. y Schwegler, A. (eds.), América negra: panorámica actual de los estudios lingüisticos sobre las variedades hispánicas, portuguesas y criollas, Frankfurt am Main, Madrid, Vervuert, Iberoamericana, pp. 139-217.

MCWhorter, J. H. (2001): «The world's simplest grammars are Creole grammars», Linguistic typology 5, pp. 125-166.

SAYCE, Ar. H. (1875/2009): The principles of comparative philology, Charleston, BiblioBazaar.

SCHLEGEL, Fr. VON (1808/1849): «On the language and wisdom of the Indians», en The aesthetic and miscellaneous works of Frederick von Schlegel, Londres, Henry G. Bohn, pp. 425-458 (trad. inglesa de Millington, E. J.). Disponible en <http://books.google.es/books?id=EF0TAAAAYAAJ\&pg=PR1\&dq=The+ aesthetic+and+miscellaneous+works+of+Frederick+von+Schlegel\&cd=1\#v= onepage\&q\&f=false $>$ (última consulta: 19-04-2010)

SмiтH, A. (1761/1853): «Considerations concerning the first formation of languages, and the different genius of original and compound languages», en The theory of moral sentiments, Londres, H. G. Bohn, pp. 505-538. Disponible en <http://books.google.es /books?id=FbYCAAAAYAAJ\&printsec=front cover\&dq $=$ The + theory + of + moral + sentiments\&cd $=1 \# \mathrm{v}=$ onepage $\& q \& f=$ false $>$ (última consulta: 17-04-2010).

Smith, Th. C. y TAPIA García, F. (2002): «El amuzgo como lengua activa», en Mevy, P. (ed.), Del cora al maya yucateco, México D. F., Universidad Nacional Autónoma de México, pp. 81-130. 
VAlDman, A. (1980): «Creolization and second acquisition», en Valdman, A. y Highfield, A. (eds.), Theoretical orientations in Creole studies, Nueva York, Academic Press, pp. 287-311.

VAN VALIN, R. D. (2007): «The role and reference grammar analysis of threeplace predicates», Suvremena lingvistika 63, pp. 31-63. Disponible en <http://linguistics.buffalo.edu/people/faculty/vanvalin/rrg.html> (última consulta: 19-04-2010). 